


\title{
Se $\mathfrak{a} \mathfrak{n} \mathfrak{a} \mathfrak{a} \mathfrak{I}$ \\ $\mathfrak{S} \ddot{a} \mathfrak{m} \mathfrak{t} \mathfrak{I} \mathfrak{i}$ d e $\mathscr{W}$ e $\mathfrak{x}$ e

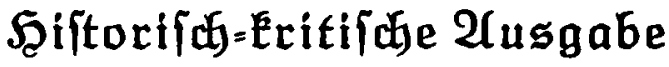

\author{
Serauggegeben \\ von \\ ber Seutfden Uabemie ber Wiffenfdhaften zu Berlin
}

Dritfe Ubfeilung

Briefe

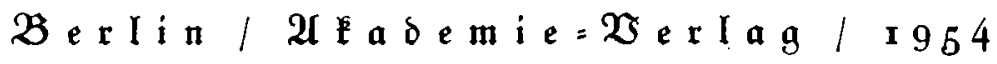




\section{$\Im \mathfrak{a} \mathfrak{n} \mathfrak{a} \mathfrak{u} \mathfrak{l} \mathfrak{s}$ \\ $\mathfrak{S} \ddot{a} \mathfrak{m} \mathfrak{t} \mathfrak{I}$ che $\mathfrak{W}$ e $\mathfrak{x}$ e

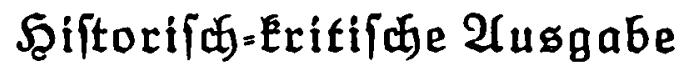

Driffe $\mathfrak{A b}$ feilung

Siebenter $\mathfrak{B a n d}$

Briefe I8I5-I8r9

Mit 6 Tafelbeilagen

Şerausgegeber

$\mathfrak{v o n}$

Euard Berend

$\mathfrak{B} \mathfrak{e} \mathfrak{r} \mathfrak{i} \mathfrak{n} / \mathfrak{Z} \mathfrak{k} \mathfrak{a} \mathfrak{e} \mathfrak{m} \mathfrak{i} \mathfrak{e}=\mathfrak{W} \mathfrak{e} \mathfrak{r} \mathfrak{a} \mathfrak{g} /$ I 954 
Copyright 1954 by áabemie= Ulle Redte vorbebalten

Erfofenen im Alabemie=-2erlag Gmb5., Berlin W 8, Mabrentraßze 39 $\mathcal{E}$ izeng=शr. $202 \cdot 1007552$

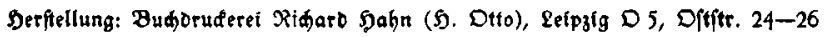

Beftell= uno Betlagsnummer $3005 / 7$

Brinteb in Germany 